$\mathrm{DTP} / 99 / 104$

hep-ph/9912206

\title{
Results in next-to-leading-log prompt-photon hadroproduction $\pitchfork$
}

\author{
Carlo Oleari \\ Department of Physics, University of Durham, Durham, DH1 3LE, UK
}

\begin{abstract}
We present some results on renormalization and factorization scale dependence of soft-gluon resummation, at the next-to-leading-logarithmic level, in the fixed-target hadro-production cross-section for prompt photons.

PACS numbers: 12.38.-t, 12,38.Cy
\end{abstract}

\section{Introduction}

The phenomenological interest in prompt-photon production in fixed-target experiments resides mainly in its use as a gluon probe in structure-function studies. Prompt-photon production is historically our main source of information on the gluon parton density at large $x$ (e.g. $x>0.2$ ). This same region is relevant for hadron colliders in production phenomena at very large transverse momenta, and thus its understanding is crucial in order to disentangle possible new physics signals from the QCD background.

In this talk, we consider the effect of soft-gluon resummation in prompt-photon production [1, 2] near the "threshold" limit, that is to say for high transverse momentum. A more extensive treatment of this subject can be found in Ref. [3].

\section{Kinematics, notation and NLL resummed cross section}

We consider the inclusive production of a single prompt photon in hadron collisions

$$
H_{1}\left(P_{1}\right)+H_{2}\left(P_{2}\right) \rightarrow \gamma(p)+X .
$$

The colliding hadrons $H_{1}$ and $H_{2}$ carry momenta $P_{1}$ and $P_{2}$, and the centre-of-mass energy squared is then given by $S=\left(P_{1}+P_{2}\right)^{2}$, while the photon momentum is $p$. If $\theta$ is the angle that the photon forms with respect to the incoming beams in the centre-of-mass frame then we define $x_{T}$ and the rapidity $y$ as

$$
x_{T}=\frac{2 E_{T}}{\sqrt{S}}, \quad y=-\log \tan \frac{\theta}{2}, \quad \text { where } \quad E_{T}=|\mathbf{p}| \sin \theta
$$

$\dagger$ Talk given at the UK Phenomenology Workshop on Collider Physics, Durham, UK, 19-24 September 1999. To appear in the Proceeding of the conference. 
Prompt-photon production takes place both by hard-photon emission from initialor final-state quarks (direct component), and by collinear radiation from final-state partons. This last mechanism is not fully calculable in perturbation theory and, in fact, it depends upon the photon fragmentation function. However, in the cases of practical interest, the corrections due to the fragmentation processes are small, and we shall limit our considerations to the hard-photon part. In this approximation, the differential prompt-photon production cross section integrated over $y$ is given by the following factorization formula

$$
\begin{aligned}
\frac{d \sigma_{\gamma}\left(x_{T}, E_{T}\right)}{d E_{T}} & =\frac{1}{E_{T}^{3}} \sum_{a, b} \int_{0}^{1} d x_{1} f_{a / H_{1}}\left(x_{1}, \mu_{F}^{2}\right) \int_{0}^{1} d x_{2} f_{b / H_{2}}\left(x_{2}, \mu_{F}^{2}\right) \\
& \times \int_{0}^{1} d x \delta\left(x-\frac{x_{T}}{\sqrt{x_{1} x_{2}}}\right) \hat{\sigma}_{a b \rightarrow \gamma}\left(x, \alpha_{s}\left(\mu^{2}\right) ; E_{T}^{2}, \mu^{2}, \mu_{F}^{2}\right) .
\end{aligned}
$$

where $a, b, c$ denotes the parton indices $(a=q, \bar{q}, g)$, and $f_{a, b / H}\left(x, \mu_{F}^{2}\right)$ are the parton densities of the colliding hadrons, evaluated at the factorization scale $\mu_{F}$. The rescaled partonic cross sections $\hat{\sigma}_{a b \rightarrow \gamma} \equiv E_{T}^{3} d \hat{\sigma}_{a b \rightarrow \gamma} / d E_{T}$ are computable in perturbative QCD as power series in the running coupling $\alpha_{s}\left(\mu^{2}\right), \mu$ being the renormalization scale in the $\overline{\mathrm{MS}}$ renormalization scheme. Their analytic expression is known till order $\alpha_{s}^{2}$ [4].

At leading order, only three subprocesses contribute to the direct-photon production

$$
q+\bar{q} \rightarrow g+\gamma, \quad q+g \rightarrow q+\gamma, \quad \bar{q}+g \rightarrow \bar{q}+\gamma .
$$

Near the partonic threshold region $x \rightarrow 1$, i.e. when $E_{T}$ reaches its maximum value, the partonic cross section are enhanced by double-logarithmic corrections, due to softgluon-radiation suppression

$$
\hat{\sigma}^{(n)}(x) \sim \hat{\sigma}^{(0)}(x)\left[a_{n, 2 n} \ln ^{2 n}(1-x)+a_{n, 2 n-1} \ln ^{2 n-1}(1-x)+\ldots\right] .
$$

Resummation of these soft-gluon effects to all orders in perturbation theory can be important to improve the reliability of the QCD predictions.

The resummation program of soft-gluon contributions has been carried out in the Mellin-transform space, or $N$-space. Working in $N$-space, we can disentangle the softgluon effects in the parton densities from those in the partonic cross section and we can straightforwardly implement and factorize the kinematic constraints of energy and longitudinal-momentum conservation. The $N$-moments are defined as follows

$$
\sigma_{\gamma, N}\left(E_{T}\right) \equiv \int_{0}^{1} d x_{T}^{2}\left(x_{T}^{2}\right)^{N-1} E_{T}^{3} \frac{d \sigma_{\gamma}\left(x_{T}, E_{T}\right)}{d E_{T}},
$$

and, in $N$-space, Eq. (3) takes a simple factorized form

$\sigma_{\gamma, N}\left(E_{T}\right)=\sum_{a, b} f_{a / H_{1}, N+1}\left(\mu_{F}^{2}\right) f_{b / H_{2}, N+1}\left(\mu_{F}^{2}\right) \hat{\sigma}_{a b \rightarrow \gamma, N}\left(\alpha_{s}\left(\mu^{2}\right) ; E_{T}^{2}, \mu^{2}, \mu_{F}^{2}\right)$.

The threshold region $x_{T} \rightarrow 1$ corresponds to the limit $N \rightarrow \infty$ in $N$-space. In this limit, a hierarchy in $\ln N$ appears and the partonic cross sections can be rewritten as

$$
\hat{\sigma}_{a b \rightarrow \gamma, N}=\hat{\sigma}_{a b \rightarrow \gamma, N}^{(\mathrm{res})}\left[1+\mathcal{O}\left(\alpha_{s} / N\right)\right], \quad a b=q \bar{q}, q g, \bar{q} g
$$


where $\mathcal{O}\left(\alpha_{s} / N\right)$ denotes terms that contribute beyond LO and are furthermore suppressed by a relative factor $\mathcal{O}(1 / N)$ at large $N$. The all-order resummation formulae can be written as

$$
\hat{\sigma}_{a b \rightarrow \gamma, N}^{(\mathrm{res})}=\alpha \alpha_{s} \hat{\sigma}_{a b \rightarrow d \gamma, N}^{(0)} C_{a b \rightarrow \gamma} \Delta_{N+1}^{a b \rightarrow d \gamma},
$$

where we dropped all the scale dependences, for ease of notation, and we define

$\Delta_{N}^{a b \rightarrow d \gamma}=\exp \left\{\ln N g_{a b}^{(1)}\left(b_{0} \alpha_{s} \ln N\right)+g_{a b}^{(2)}\left(b_{0} \alpha_{s} \ln N\right)+\mathcal{O}\left(\alpha_{s}\left(\alpha_{s} \ln N\right)^{k}\right)\right\}$,

$C_{a b \rightarrow \gamma}=1+\sum_{n=1}^{+\infty}\left(\frac{\alpha_{s}}{\pi}\right)^{n} C_{a b \rightarrow \gamma}^{(n)}=1+\frac{\alpha_{s}}{\pi} C_{a b \rightarrow \gamma}^{(1)}+\mathcal{O}\left(\alpha_{s}^{2}\right)$,

$b_{0}$ being the first coefficient of the QCD $\beta$-function. The function $\ln N g_{a b}^{(1)}$ resums all the leading logarithmic (LL) contributions $\alpha_{s}^{n} \ln ^{n+1} N$ in the exponent, $g_{a b}^{(2)}$ contains the next-to-leading logarithmic (NLL) terms $\alpha_{s}^{n} \ln ^{n} N$, and so forth. The analytic expressions for the functions $g_{a b}^{(1)}, g_{a b}^{(2)}$ and $C_{a b \rightarrow \gamma}^{(1)}$ have been computed in Ref. [2].

\section{Hadron-level results}

The main points we intend to highlight for the full hadronic cross sections are:

(i) the size of the NLL corrections, relative to the NLO contributions;

(ii) the scale dependence at NLL order.

The size and scale dependence of the resummed cross section $(q g+q \bar{q}$ contributions), compared to the NLO one, is given in Fig. 1. We plot the distributions as a function of the beam energy $\left(E_{\text {beam }}\right)$ for the fixed value of $E_{T}=10 \mathrm{GeV}$. Different values of $E_{\text {beam }}$, therefore, probe different ranges of $x_{T}$, as indicated by the upper labels on the plots. Note the significant reduction in scale dependence in both cases: with and without the inclusion of the constant term $C_{a b \rightarrow \gamma}^{(1)}$.

We also explored the renormalization- and factorization-scale dependence of our calculations. The results, for $p N$ collisions at $E_{\text {beam }}=10 \mathrm{GeV}$, are shown in Fig. 2. A significant improvement in the stability of the results, relative to the dependence at LO and NLO, is observed in all cases.

\section{Acknowledgments}

The work presented here has been done in collaboration with S. Catani, M. L. Mangano, P. Nason and W. Vogelsang.

\section{References}

[1] Laenen E, Oderda G and Sterman G 1998 Phys. Lett. 438173.

[2] Catani S, Mangano M L and Nason P 1998 JHEP 9807024.

[3] Catani S, Mangano M L, Nason P, Oleari C and Vogelsang W 1999 JHEP 03025.

[4] Aurenche P, Baier R, Douiri A, Fontannaz M and Schiff D 1984 Phys. Lett. 140 87;

Aurenche P, Baier R, Fontannaz M and Schiff D 1988 Nucl. Phys. 297661. 

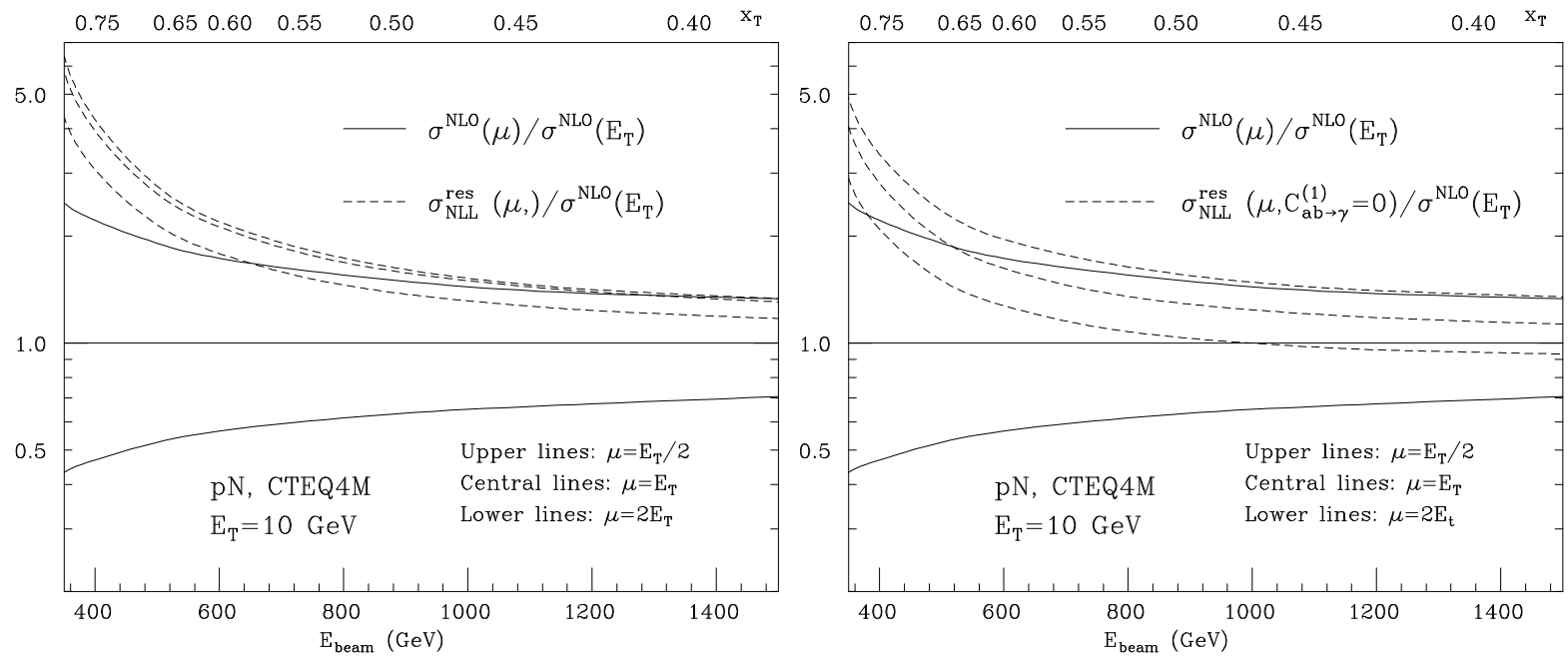

Figure 1. Relative size and scale dependence of $d \sigma / d E_{T}(q g+q \bar{q}$ components) for prompt photons in $p N$ collisions, at $E_{T}=10 \mathrm{GeV}$, plotted as a function of the protonbeam energy, $E_{\text {beam }}$ (the associated values of $x_{T}$ are given on the top scale). The solid lines represent the exact NLO result for different choices of $\mu=\mu_{F}\left(\mu=E_{T} / 2\right.$ and $2 E_{T}$ ), normalized to the $\mu=E_{T}$ result. The dashed lines represent the NLO+NLL result (in the right panel we have set $C_{a b \rightarrow \gamma}^{(1)}=0$ ) for different choices of $\mu$, normalized to the NLO $\mu=E_{T}$ result.

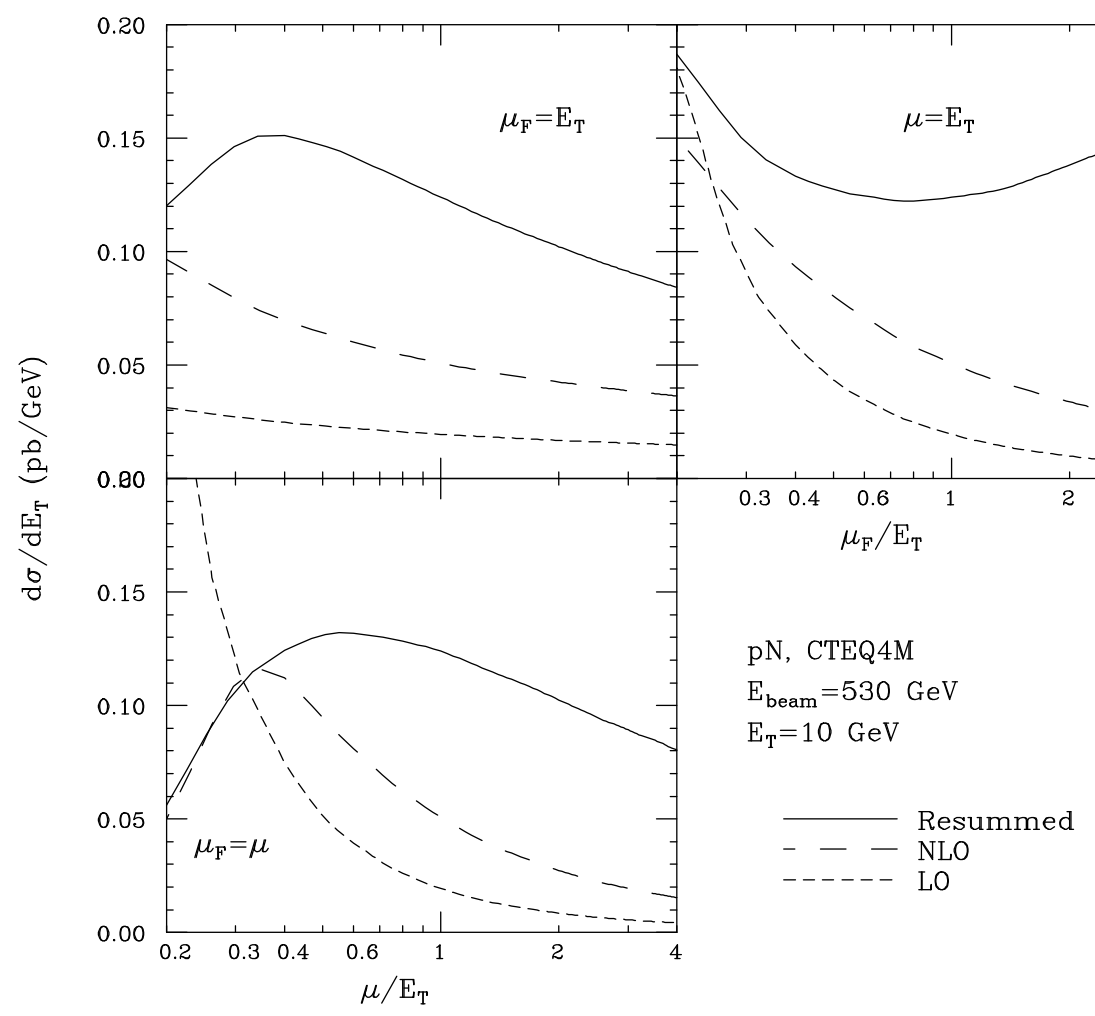

Figure 2. Scale dependence of the differential $E_{T}$ distribution in $p N$ collisions, for $E_{T}=10 \mathrm{GeV}$. We compare the results at the Born and NLO level with the results of the resummed calculation. Upper left: renormalization-scale dependence, with the factorization scale fixed to $\mu_{\mathrm{F}}=E_{T}$. Upper right: factorization-scale dependence, with the renormalization scale fixed to $\mu=E_{T}$. Lower left: scale dependence, with the renormalization and factorization scales equal. 